\title{
Captures
}

Figures, théories et pratiques de l'imaginaire

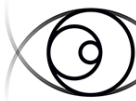

\section{Utopies et anxiétés de la photographie en régime numérique Présentation du dossier}

\section{Martha Langford and Vincent Lavoie}

Volume 1, Number 1, 2016

Post-photographie?

URI: https://id.erudit.org/iderudit/1059819ar

DOI: https://doi.org/10.7202/1059819ar

See table of contents

Publisher(s)

Figura, Centre de recherche sur le texte et l'imaginaire

ISSN

2371-1930 (digital)

Explore this journal

Cite this article

Langford, M. \& Lavoie, V. (2016). Utopies et anxiétés de la photographie en régime numérique : présentation du dossier. Captures, 1(1).

https://doi.org/10.7202/1059819ar
Article abstract

Évoquée d'abord dans les années 90 alors que la photographie numérique faisait son entrée dans les usages professionnels puis amateurs, la notion de post-photographie est récemment revenue au devant de la scène à la faveur d'expositions et de publications désireuses d'en comprendre les

déterminations esthétiques, sociales et économiques. Une mutation des usages photographiques (Facebook, Flickr, Instagram) expliquerait...
Tous droits réservés (c) Martha Langford, Vincent Lavoie, 2016

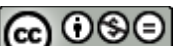

This document is protected by copyright law. Use of the services of Érudit (including reproduction) is subject to its terms and conditions, which can be viewed online.

https://apropos.erudit.org/en/users/policy-on-use/ 
Version enrichie de cet article : http://revuecaptures.org/node/418

\section{Utopies et anxiétés de la photographie en régime numérique}

\section{Présentation du dossier}

\section{Martha Langford \\ Vincent Lavoie}

Résumé :

Évoquée d'abord dans les années 90 alors que la photographie numérique faisait son entrée dans les usages professionnels puis amateurs, la notion de post-photographie est récemment revenue au devant de la scène à la faveur d'expositions et de publications désireuses d'en comprendre les déterminations esthétiques, sociales et économiques. Une mutation des usages photographiques (Facebook, Flickr, Instagram) expliquerait...

Évoquée pour la première fois dans les années 90 alors que la photographie numérique faisait son entrée dans les usages professionnels puis amateurs, la notion de post-photographie est récemment revenue au devant de la scène à la faveur d'expositions et de publications désireuses d'en comprendre les déterminations esthétiques, sociales et économiques. Une mutation des usages photographiques notamment attribuable au développement de la téléphonie mobile et à la création de plateformes de partage (Facebook, Flickr, Instagram) expliquerait ce regain d'intérêt pour une notion désormais rattachée à cette " seconde révolution numérique » actuellement en cours. Jadis formulée dans la foulée des théories post-modernistes, la postphotographie caractériserait aujourd'hui l'ensemble des pratiques, amateur ou non, de l'actuel régime numérique de production et de diffusion des images. Massification de la production photographique, présence ubiquitaire des contenus visuels, instauration de nouveaux modes de socialisation autour de l'image numérique, photojournalisme citoyen 1 , mais également dématérialisation de l'image photographique, banalisation de ses emplois et démultiplication de ses réemplois figurent au nombre des traits propres à cette seconde révolution. Et comme toute révolution, celle-ci génère des utopies - nouvelles communautés virtuelles, triomphe de l'image conversationnelle, célébration du partage, communion globale au prétexte de l'image - et bien entendu des angoisses : impuissance devant le flux incontrôlé de la production visuelle, thésaurisation impossible des représentations visuelles, perte définitive de la croyance en la véracité des images, etc. Si ces considérations touchent principalement les usages sociaux de la photographie numérique, celles-ci affectent également la création photographique contemporaine qui s'en empare et les métabolise pour en proposer une réponse artistique. Car la redéfinition de certaines conduites sociales vis-à-vis de la photographie engage une interrogation artistique de ces mêmes conduites : recours massif aux procédures d'appropriation, redéfinition de la notion d'auteur, amateurisation des protocoles figuratifs, dématérialisation de 
l'objet d'art, etc. Si ces interrogations ne sont pas nouvelles, elles sont reformulées à l'aulne de la nouvelle donne numérique.

C'est à l'analyse de ces utopies et anxiétés de la photographie en régime numérique, et de leur traduction artistique, que le présent dossier est consacré. Issues pour l'essentiel du colloque « À partir d'aujourd'hui... Reconsidering Postphotography 2 », tenu à l'automne 2015 dans le cadre du Mois de la Photo à Montréal et dont la thématique à l'honneur était « La condition post-photographique », les contributions de ce dossier réévaluent les prémisses de cette notion, en nuancent la force de rupture, l'inscrivent dans une histoire longue, en exposent les apories et la part fantasmatique, selon des perspectives disciplinaires variées. Car le phénomène en question est irréductible à un seul domaine de compétences. C'est ainsi que les pratiques visuelles conversationnelles entre adolescents se prêtent, comme en témoigne la contribution de Jocelyn Lachance, à une analyse sociologique interrogeant la valeur communicationnelle des images mises en ligne par ceux-ci sur les diverses plates-formes de partage. Mais par-delà la dimension transactionnelle associée à la diffusion publique des imageries de soi, la production photographique parfois compulsive des adolescents traduirait l'intention sinon de maitriser le temps par l'image, du moins de produire un temps commun partagé par l'ensemble des interlocuteurs.

Le temps de l'image numérique en régime post-photographique se déploie horizontalement, ainsi que l'illustre la médiatisation virale de certaines outrances planétaires, telles que celle déclenchée par l'abattage par un dentiste américain, chasseur amateur de grand gibier, d'un lion prénommé Cecil, le 1er juillet 2015. L'étude de Karla McManus expose le rôle joué par la photographie et le paratexte - commentaires des internautes, légendes, titraille, etc. - dans la fabrication d'une indignation transnationale. McManus reprend la chronologie des faits et retrace les épisodes d'une vindicte citoyenne et militante alimentée par le recyclage intensif d'une iconographie procédant d'un imaginaire environnementaliste globalisé et mettant en exergue les iniquités économiques entre l'Afrique et les pays de l'ouest. Force est de constater que les images, même celles circulant sur le Web et dont la provenance et l'authenticité apparaissent hautement incertaines, demeurent le support des convictions et des croyances les plus diverses. La dématérialisation des images, leur dissémination souvent anarchique sur toutes les plates-formes, les circonstances souvent inconnues de leur réalisation, l'ignorance de leurs usages premiers, en somme le statut orphelin de celles-ci au regard de l'ensemble des déterminations réputées fondamentales à leur compréhension - à savoir leur cadrage historique, conceptuel, culturel, institutionnel, social ou esthétique - n'aura pas suffit à annihiler leur pouvoir de persuasion. Or, la photographie à l'ère numérique ne cesse d'être suspectée des pires tromperies. Depuis l'introduction des outils informatiques dans les rédactions à la fin des années 80 jusqu'à la vogue commerciale des applications et services de partage permettant à chacun de « customiser » à souhait ses images (Instagram, Hipstamatic, etc.), la véracité jadis attribuée aux images techniques suscitent la plus grande 
méfiance. À telle enseigne que cette suspicion contamine désormais le regard que nous portons sur l'ensemble des images, même celles d'avant le numérique. La photographie ouvrirait à un regard paranoïaque, comme le constate Maxime Coulombe en observant les arguments invoqués dès 1994 par William J. Mitchell. C'est par le truchement d'un fascinant jeu de miroir entre W. J. Mitchell, auteur de The Reconfigured Eye, ce livre phare de la post-photographie, et son homonyme W. J. T. Mitchell, fondateur du pictorial turn, que Coulombe analyse la complexité de ce doute dont la photographie est frappée. En tissant un lien historique et conceptuel entre ces deux figures, Coulombe repose la question de la ressemblance, de l'indicialité et de la conventionnalité de la photographie, resituant ainsi les images rattachées au moment post-photographique dans l'arène d'un débat épistémologique au long cours. Inscrire la post-photographie dans le cadre d'un questionnement théorique et philosophique élargi est également ce qui anime Daniel Fiset, dont la contribution invite à nuancer la valeur de rupture communément associée à la photographie numérique. Sortir la postphotographie du présentisme (Hartog) dont elle serait devenue le symbole, historiciser les discours actuellement tenus sur cette photographie de l'« après-photographie », comprendre cette crise du réel attribuable à la dissolution des images dans l'espace-Web, voilà quelques-unes des ambitions de cet article, que son auteur cheville à la pensée heideggérienne sur la technique. Une technique qui se veut invisible, intangible, mais surtout " bunkerisée » au sein de centres de données hautement sécurisés, ainsi que l'analyse Nina Lager Vestberg dans un article s'intéressant à l'implantation de ces infrastructres en Europe du Nord. Énergivores, ces installations technologiques de pointe se multiplient dans les pays tempérés, non sans laisser une empreinte écologique. Par sa contribution, Vestberg entend rappeler que la postphotographie, aussi impalpable qu'elle puisse parfois paraître, pèse de tout son poids dans le bilan carbone global.

C'est là l'un des fils rouges parcourant plusieurs contributions de ce dossier : la post-photographie ne représente pas tant un régime visuel nouveau qu'une reprise sous le mode de l'exacerbation de conduites et de postures historiques. Suzanne Paquet en produit une éloquente démonstration en relevant dans les pratiques post-photographiques des amateurs et des artistes contemporains des traits et procédures rappelant ceux des photographes du XIX`e siècle. Les « Esquisses physiologiques » (1852-53) d’Ernest Lacan, cette série de textes dans lesquels le rédacteur en chef de la revue française La Lumière dresse la typologie des praticiens de la photographie - le photographe proprement dit, le photographe artiste, le photographe amateur et le photographe savant -, forment le socle d'une analyse posant les fondements d'une protohistoire de la post-photographie. L'historicisation de la post-photographie est également centrale à la contribution de Ji-Yoon Han, où celle-ci s'emploie à analyser les points de convergence entre les pratiques contemporaines rattachées à cette mouvance et la photographie surréaliste. Que ce soit au plan de l'iconographie, de la compulsion à la thésaurisation, de la décontextualisation des images ou encore des protocoles de sélection - par automatisme jadis, par algorithmes aujourd'hui - la post-photographie procèderait de l'après-coup, au sens freudien du 
terme, d'un rapport à la photographie institué plus tôt dans le siècle dernier. Mais par-delà les correspondances historiques finement relevées par l'auteure, Han interroge la nature de l'éclairage que la post-photographie est à même de jeter sur l'histoire du médium photographique. Enfin, Annebella Pollen, à l'instar de plusieurs auteurs participant à ce dossier, nuance le caractère de nouveauté communément attribué à la post-photographie en rappelant à la mémoire des projets plus anciens de massification de la photographie amateur, dont certains datent de la fin des années 80 , soit quelques années avant la première révolution numérique introduite par la commercialisation des premiers appareils photographiques non argentiques. L'analyse de ces compilations de clichés amateur conduit Pollen à remettre en cause les discours ne voyant dans la post-photographie qu'un torrent d'images indifférenciées. Ayant interrogé les auteurs de ces clichés sur leur motivation et sur leurs choix iconographiques, Pollen rappelle à raison que toute photographie, aussi banale soit-elle, procède d'un acte pourvu d'affect - preuve en sont les images de chatons qui inondent aujourd'hui le Web - qu'aucune opération de massification m'annihilera totalement. Car tel est certainement l'autre dénominateur commun à l'ensemble des articles réunis dans ce dossier : la prise en compte de l'irréductible charge affective dont les images sont porteuses, que ce soit à travers les indignations dont elles sont les agents, les plaisirs qu'elles procurent, les curiosités qu'elles alimentent ou les anxiétés qu'elles génèrent par leur incoercible prolifération.

1. Citons à titre d'exemple le rôle joué par la photographie mobile dans la production et la diffusion des images de la Révolution verte en Iran. Voir James, Henry et Natalia Lebedinskala. 2012. " Green Photographs: An In-Moving Memory », in Imaginaires du présent. Photographie, politique et poétique de l'actualité. Cahier ReMix, $\mathrm{n}^{\circ} 1$ (mai 2012), Montréal, Figura, Centre de recherche sur le texte et l'imaginaire. En ligne.

2. Le comité scientifique de ce colloque organisé conjointement par l'Université Concordia (UC), l'Université du Québec à Montréal (UQAM) et le Mois de la Photo à Montréal (MPM) était composé de Joan Fontcuberta (MPM), Corina llea (MPM), Samuel Gaudreau-Lalande (UC), Martha Langford (UC), Julie-Ann Latulippe (UQAM) et Vincent Lavoie (UQAM). 